\title{
Nonlinear Correction of Sensor Based on Immunization Programs
}

\author{
Lirong $\mathrm{Lu}^{1}$, Xiaodong $\mathrm{Niu}^{2}$, Jinyang $\mathrm{Zhou}^{1, *}$ and Chunhua Shi ${ }^{1}$ \\ ${ }^{I}$ Biomedical Engineering, Changzhi Medical College, Changzhi, Shanxi, 046000, China \\ ${ }^{2}$ Department of Physics, Changzhi Medical College, Changzhi, Shanxi, 046000, China
}

\begin{abstract}
Sensor applications in medical instrumentation measurement are very extensive, but mostly the input and output of the sensor is nonlinear, which brings a great inconvenience for the measure of medical instruments. This paper introduces biological immune system on the basis of the evolution planning, thereby forming immunization programs to use non-linear calibration of the sensor. This method joins nonlinear correction links after sensor amplification and A/D converter links and uses the immunization program to obtain the polynomial coefficients of the nonlinear correction link. Simulations show that using immunization programs to have non-linear calibration to sensor, gives high correction accuracy, faster convergence speed and very good stability.
\end{abstract}

Keywords: Immunization programs, non-linear calibration, sensor.

\section{INTRODUCTION}

Sensor [1] is a device which can convert measurement into the power output. Sensor plays an important role in the development of medical instruments and medical experiments. In the ideal state, there is a linear relationship between the input and output of the sensor, by measuring the amount of the output, we can find the input size. However, in practical applications, most of the relationship between sensor output and input is non-linear. To solve this problem, people use many methods to correct the non-linearity of sensor. However, these corrective methods at this stage exist with defects such as high cost, poor accuracy and poor stability $[2,3]$.

Evolutionary Programming [4] which is an evolutionary algorithm originated in the $1960 \mathrm{~s}$ is a random search algorithm obtained by simulating the process of natural evolution. In evolutionary plan, use of the cross and reorganization operator is not recommended, which can reflect the individual interplay. However, only use of mutation operator such as Gaussian mutation operator is suggested in most cases, because its algorithm is simple. Mutation operator can modify an individual with a certain probability, but maybe the mutation individual is not suitable as a parent, which can be concluded as there being a degradation. All practical problems themselves have certain characteristics, using these features it will improve solving speed, but evolutionary programming doesn't take advantage of these features. The two points above influence the running speed of the algorithm. The ability to maintain evolutionary programming solutions group diversity is insufficient, and easy to premature convergence [5].

Biological immune system [6] is an important system of the body to perform an immune response and immune

\footnotetext{
*Address correspondence to this author at the Biomedical Engineering, Changzhi Medical College, Changzhi, Shanxi, 046000, China; Tel: 15235592783; E-mail: 1lr1982@163.com
}

function, it can recognize self and others and rapidly remove and eliminate foreign bodies to ensure the safety of the body. Biological immune system has functions of producing a variety of antibodies, self-regulation and immune memory. The idea of this system's work is applied in engineering practice, such as the attitude control of satellite; for more details refer to the literature [7]. Diagnosis of RNA-based viruses sometimes needs the help of nonlinear correction [8], so the method must undergo further research to find more benefits for the development of the society.

Apply the mechanism of biological immune system to evolutionary programming algorithm by drawing the ability of immune system to produce and maintain the diversity of antibodies and self-regulation. In the overall framework of evolutionary algorithms, immune mechanism is introduced to form immunization program (referred to as IP). This paper studies the application of the immunization program to solve non-linear calibration of sensor system. Experimental results show that application of the immunization program for nonlinear calibration can not only improve the correction accuracy, but also greatly improve the speed and stability.

\section{CORRECTION MODEL}

The correction model is shown in Fig. (1).

In Fig. (1), $X$ is the measured variable as the input signal of the sensor. After the sensor, $X$ becomes a non-linear output signal, then after amplification and A/D converter links, the output becomes $\mathrm{Y}$, which is also non-linear with $\mathrm{X}$, finally after IP correction, $\mathrm{Z}$ becomes the output.

IP correction mainly performs data processing and nonlinear correction to make sure that the relationship between the final output $\mathrm{Z}$ and input of sensor $\mathrm{X}$ is linear. 


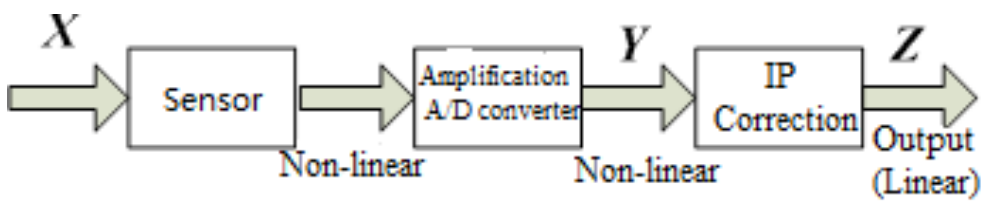

Fig. (1). Correction model.

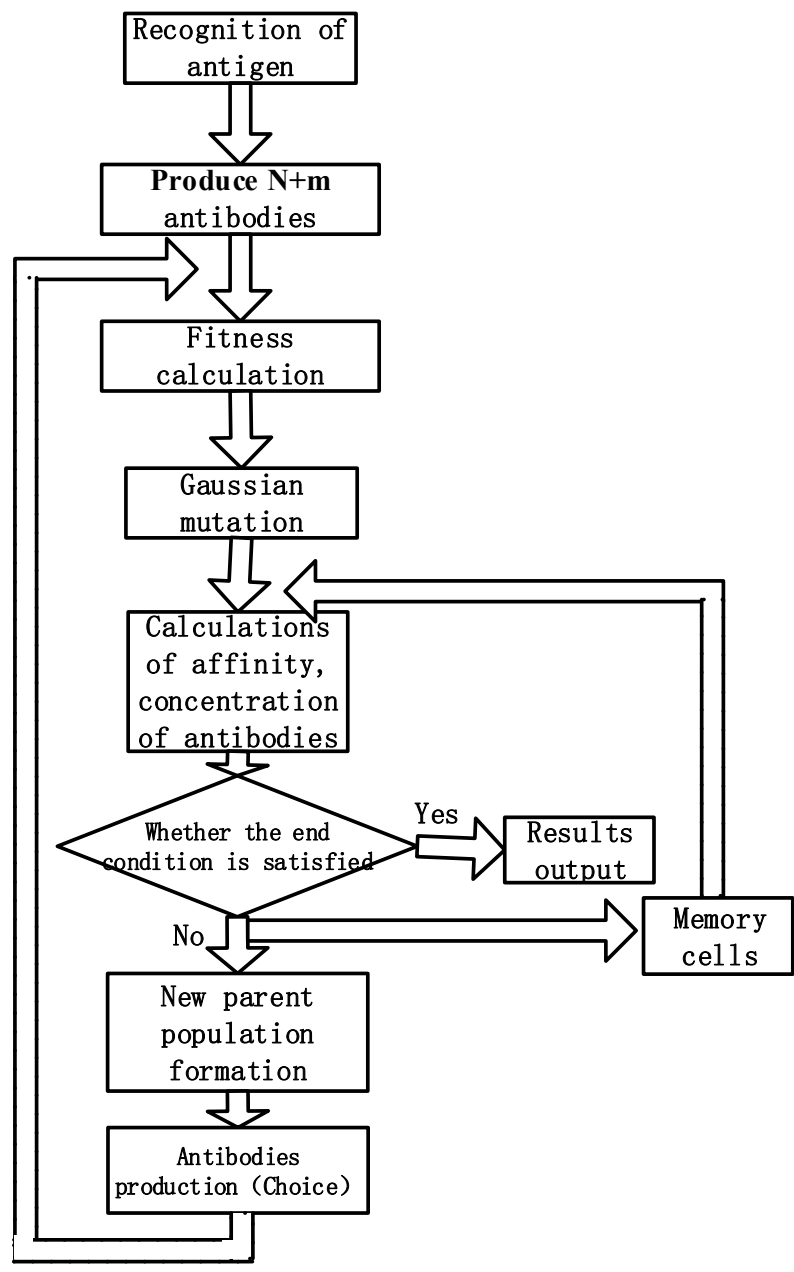

Fig. (2). IP flow chart.

Given the relationship between $Y$ and $X$ is: $Y=f(X)$, that between $Z$ and $Y$ is: $Z=g(Y)$, if $g(Y)=f^{-1}(Y)$, then

$$
Z=g(Y)=f^{-1}(Y)=X
$$

As we can see, the whole circuit output $\mathrm{Z}$ and its input $\mathrm{X}$ is equal, which is certainly linear, namely, non-linear correction succeeds. The purpose of this paper is to use IP to seek correction link $Z=g(Y)$, namely, $Z=f^{-1}(Y)$.

The variable $Z$ can be expressed as:

$$
Z=f^{-1}(Y)=a_{0}+a_{1} Y+a_{2} Y^{2}+\ldots+a_{n} Y^{n}
$$

Where, $a_{0}, a_{1}, a_{2}, \ldots ., a_{n}$ are coefficients to be determined, the value of $n$ is determined by required accuracy. In this paper, $n=3$, namely
$Z=f^{-1}(Y)=a_{0}+a_{1} Y+a_{2} Y^{2}+a_{3} Y^{3}$

Where, $a_{0}, a_{1}, a_{2}, a_{3}$ are coefficients to be determined, when coefficients in (3) are determined, the correction link $Z=f^{-1}(Y)$ is also determined.

\section{STEPS OF USING IP TO IMPLEMENT NON- LINEARITY CORRECTION OF SENSOR}

By applying the mechanism of biological immune system to evolutionary programming algorithms, immunization programs are formed. IP flow chart is shown in Fig. (2).

The steps of using IP to solve the nonlinear correction problem of sensor system are as follows: 
Table 1. Sensor output $\boldsymbol{F}$ and corresponding actual input concentration value $\boldsymbol{C p}$.

\begin{tabular}{|c|c|c|c|c|c|c|c|c|}
\hline $\mathbf{F ~ ( H Z ) ~}$ & $\mathbf{2 5 0}$ & $\mathbf{5 0 0}$ & $\mathbf{7 5 0}$ & $\mathbf{1 0 0 0}$ & $\mathbf{1 2 5 0}$ & $\mathbf{1 5 0 0}$ & $\mathbf{1 7 5 0}$ & $\mathbf{2 0 0 0}$ \\
\hline \hline $\mathrm{Cp}(\%)$ & 5.06 & 4.32 & 3.68 & 3.37 & 2.77 & 2.36 & 2.24 & 2.09 \\
\hline
\end{tabular}

(1) Recognition of antigen is the problem to be solved. Recognition of antigen is to find the objective function corresponding to the problem. In this paper, the objective function of sensor nonlinearity correction is:

$$
f_{1}(x)=\sum_{i=1}^{n}(Z-X)^{2}=\sum_{i=1}^{n}\left(a_{0}+a_{1} Y+a_{2} Y^{2}+a_{3} Y^{3}-X\right)^{2}
$$

(2) The initial production of antibodies. We can use computer to randomly produce $\mathrm{N}+\mathrm{m}$ individuals as the initial population and put $\mathrm{m}$ individuals into memory bank.

(3) Individual fitness calculation. Individual fitness is determined by the following formula:

$$
F(x)=\frac{1}{f_{1}(x)}=\frac{1}{\sum_{i=1}^{n}(Z-X)^{2}}=\frac{1}{\sum_{i=1}^{n}\left(a_{0}+a_{1} Y+a_{2} Y^{2}+a_{3} Y^{3}-X\right)^{2}}
$$

(4) Gaussian mutation. Gauss mutation operator is mostly used in IP. In the process of mutation, calculate linear square root of each individual fitness function value to obtain the standard deviation of individual variation, each component plus a random obeying lognormal.

Given $X$ is individual, $\sigma$ is the standard deviation of Gaussian mutation. Chromosomal individual has $\mathrm{L}$ gene locations. Then, $(X, \sigma)=\left(\left(x_{1}, x_{2}, \cdots, x_{L}\right), \sigma\right)$. After Gaussian mutation,

$x_{i}(t+1)=x_{i}(t)+N(0, \sigma(t+1))$

Where,

$\sigma(t+1)=\sqrt{\beta F(X(t))+\gamma}$

Where, $N(0, \sigma(t+1))$ denotes random variables whose mean is 0 and variance is $\sigma(t+1), F(X(t))$ indicates the fitness of current individual, $\beta, \gamma$ is coefficient to be determined, usually, the value of $\beta$ and $\gamma$ is 1 and 0 respectiively.

(5) Calculation of antibody affinity and concentration. The computing object of the $\mathrm{N}$ new individuals of Gaussian mutation, and $\mathrm{m}$ individuals in memory bank.

Affinity of the antibody and antigen.

We can regard the affinity of the antibody and antigen as the match degree between the objective function and feasible solution, which can be regarded as individual fitness here. The affinity function in this paper is:

$$
\left(A_{g}\right)_{k}=\frac{1}{f_{1}(x)}=\frac{1}{\sum_{i=1}^{n}(Z-X)^{2}}=\frac{1}{\sum_{i=1}^{n}\left(a_{0}+a_{1} Y+a_{2} Y^{2}+a_{3} Y^{3}-X\right)^{2}}
$$

$\left(A_{g}\right)_{k}$ means the affinities between antibody and antigen.

Affinity of the antibody and antibody.

Affinity of the antibody and antibody is the similarity degree between antibodies [6]. In this paper, we define the affinity between antibody $x$ and antibody $y$ as:

$\left(A_{b}\right)_{x, y}=\frac{k_{x, y}}{L}$

Where, $k_{x, y}$ is the same gene location between antibody $\mathrm{x}$ and antibody $\mathrm{y}, L$ is the length of antibody $x$ and $y$.

We define the concentration of the antibody as:

$C_{V}=\frac{1}{N} \sum_{j \in N}\left(A_{b}\right)_{x, y}$

$\mathrm{N}$ is total antibodies in population. When $\left(A_{b}\right)_{x, y}>T$, $\left(A_{b}\right)_{x, y}=1$, when $\left(A_{b}\right)_{x, y} \leq T,\left(A_{b}\right)_{x, y}=0 . T$ is a constant set in advance. $C_{V}$ represents the proportion of similar antibodies in population.

(6) Conditions judge. Determine whether the termination condition is satisfied. Yes means end of the output, otherwise, continue.

(7) The formation of a new parent population. The formation of a new parent is based on an individual breeding expected probability. Simplified desired individual probability propagation can be calculated [9] as:

$P=\frac{\left(A_{g}\right)_{k}}{C_{V}}$

As is seen, $\left(A_{g}\right)_{k}$ is higher, $P$ is bigger. $C V$ is bigger, $P$ is smaller. Namely, we hope for the high-fitness and lowconcentration individual to breed to maintain the diversity of population.

(8) New antibodies production. In accordance with the roulette wheel selection mechanism to make selection operation, the selected probability of an individual is the individual desired reproduction with formula (11) to calculate.

(9) Turn to step (3)

\section{ANALYSIS OF CASE}

In order to verify the nature of the proposed method, we have done a lot in the MATLAB simulation. The input and output data is from concentration sensor in [10]. The variable $\mathrm{f}$ in Table 1 means the output of sensor; $C \mathrm{p}$ means the input of the sensor. 


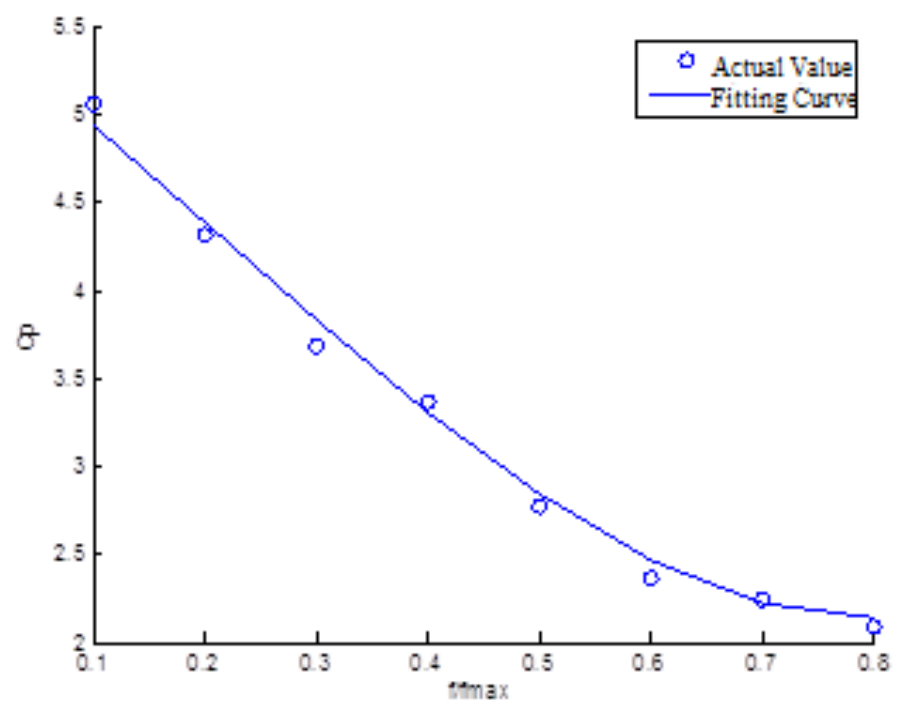

Fig. (3). Actual value and ip fitting curve.

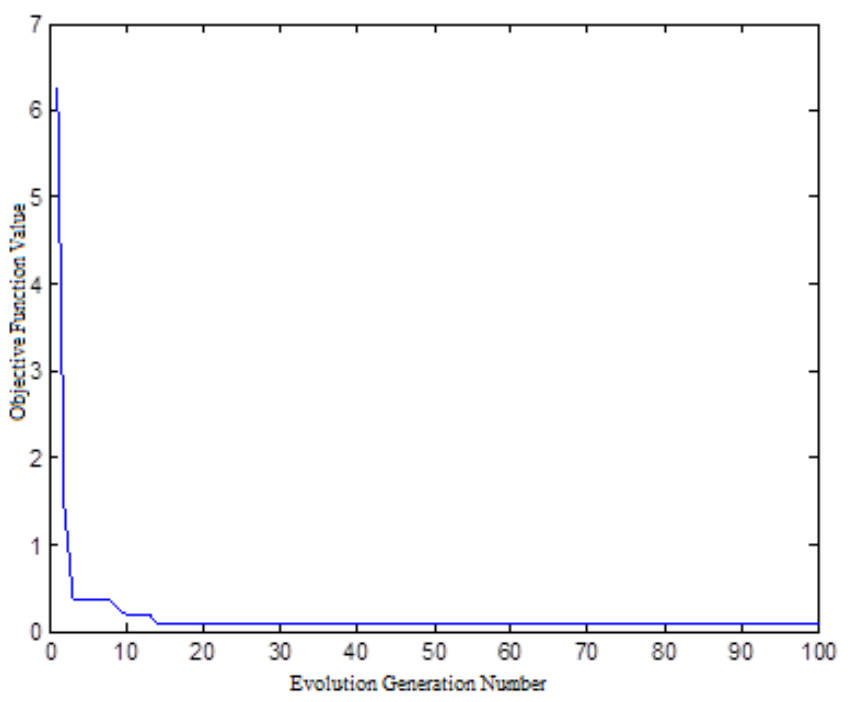

Fig. (4). The optimal value change after 100 iterations.

From the table, it is shown the relationship between the output $f$ and corresponding practical input $s$ concentration value $C \mathrm{p}$ is non-linear. Here, we must add non-linear correction link in the output of the sensor, so that there is a linear relationship between the final output and input.

Correction link

$$
\begin{aligned}
& Z=f^{-1}(Y)=a_{0}+a_{1} Y+a_{2} Y^{2}+a_{3} Y^{3} \\
& =a_{0}+a_{1} \frac{f}{f_{\max }}+a_{2}\left(\frac{f}{f_{\max }}\right)^{2}+a_{3}\left(\frac{f}{f_{\max }}\right)^{3}
\end{aligned}
$$

Where, $f \max =2500 \mathrm{HZ}, a_{0}, a_{1}, a_{2}, a_{3}$ are coefficients to be determined. The paper uses IP to determine the polynomial coefficients of non-linear correction link. We take iterations as 100 , the median of $a_{0}, a_{1}, a_{2}, a_{3}$ is 20 , individual number is 50 , and memory bank is 10 .
Operate IP and the results can be seen as follows:

Fig. (3) shows the actual input concentration and contrast value of IP fitting curve. Circles represent the actual output frequency corresponding to the actual input concentration. The solid line represents the output of the non-corrected link corresponding to the actual input frequency in non-linear correction link as shown by Fig. (3): corresponding to the same frequency, the actual value of the input density and the output value after non-linear correction is essentially the same, namely, the input and output of the entire concentration sensor system is substantially the same, in order to achieve the linear output and a non-linear calibration of concentration sensor.

Fig. (4) is the optimal solution change figure of using IP for nonlinear correction after 100 iterations, the horizontal axis represents the evolution generation, and the vertical axis represents the objective function value. The smaller the target function value, the better the non-linear correction. As 
we can see, population converges to the minimum after 58 evolve generation. The minimum value of the objective function is 0.0635 and available coefficients to be determined are

$$
a_{0}=5.4478, a_{1}=-4.8007, a_{2}=-3.5829, a_{3}=5.5091
$$

Namely,

$$
Z=5.4478-4.8007 \frac{f}{f_{\max }}-3.5829\left(\frac{f}{f_{\max }}\right)^{2}+5.5091\left(\frac{f}{f_{\max }}\right)^{3}
$$

With examples of the analysis, it can be seen that the use of IP for non-linear correction of sensor, the input and output of an entire concentration sensor is basically the same and objective function value may reach 0.0635 , indicating that this correction method has a higher precision accuracy. In addition, curve converges can also be seen to the minimum of the objective function after 58 generation, indicating that this correction method has a faster convergence rate. Running the program 100 times, $99 \%$ can converge to the minimum value of the objective function, indicating that this correction method has a very good stability.

\section{CONCLUSION}

As the core component of medical measurements, the accuracy and stability of sensor will directly affect the accuracy and stability of the entire medical measurements. Since most sensors's input and output consists non-linear characteristics, therefore they bring much inconvenience to the medical equipment measuring. This paper introduces the biological immune system based evolutionary programming, thus forming immunization programs, after amplifying circuit and A/D converter circuit, non-linearity correction link is introduced, and use the immunization program to obtain non-linear calibration coefficients of the polynomial links. Thus, we can conclude that the relationship between output and input is linear, i.e., we will achieve non-linear calibration of sensor. Finally, we use the IP to obtain a non-linear calibration of concentration sensor in the literature [8]. Practice shows that the use of IP for non-linear calibration has a high calibration accuracy, faster convergence rate and very good stability.

\section{CONFLICT OF INTEREST}

The authors confirm that this article content has no conflict of interest.

\section{ACKNOWLEDGEMENTS}

This work is supported by Research and Development Project Fund of University Technology in Shanxi (No. 20091025).

\section{REFERENCES}

[1] A. Chen, Medical Sensor, $2^{\text {nd }}$ ed. Science Press: Beijing, vol. 1, 2008.

[2] J. Liu, "Nonlinear correction method of sensor based on annealing genetic algorithm," Transducer and Microsystem Technologies, vol. 30, pp. 58-60, 2011.

[3] C. Liu, F. Wang, K. Shi, X. Wang, and Z. Sun, "Robust Hळ control for satellite attitude control system with uncertainties and additive perturbation," International Journal of Science, vol. 1, pp. 1-9, 2014.

[4] T. Liu, and H. Wang, "Genetic support vector method of nonlinear correction of sensor," Journal of Electronic Measurement and Instrument, vol. 25, pp. 56-60, 2011.

[5] D. Chen, and C. Zhao, "An improved adaptive immune evolutionary programming method and its application," Journal of System Simulation, vol. 18, pp. 1147-1150, 2006.

[6] F. Shi, and H. Wang, "30 Analysis Cases in MATLAB Intelligent Algorithms,” Beihang University Press, 2011, pp. 118.

[7] M. Zhou, and S. Sun, "Principle and application of genetic algorithm," National Defense Industry Press, pp. 32-58, 1999.

[8] C. Liao, G. Lee, and H. Liu, T. Hsieh, and C.H. Luo "Miniature RT-PCR system for diagnosis of RNA-based viruses," Nucleic acids Research, vol. 33, p. 156, 2005.

[9] X.L. Xu, W. Wang, and Q. Guan, "Adaptive Immune Algorithm for Solving Job-Shop Scheduling Problem," Springer-Verlag: Berlin Heidelberg, 2005.

[10] Y. Shen, "A new method for pulp concentration sensor calibration and dynamic nonlinear estimation," Chinese Journal of Scientific Instrument, vol. 18, pp. 1-6, 1997.

(C) Lu et al.; Licensee Bentham Open.

This is an open access article licensed under the terms of the Creative Commons Attribution Non-Commercial License (http://creativecommons.org/licenses/by-nc/4.0/) which permits unrestricted, non-commercial use, distribution and reproduction in any medium, provided the work is properly cited. 